\title{
El concepto de cultura del derecho de autor
}

Fernando Charria-García*

\footnotetext{
Doctor (c) en Derecho y Ciencias Sociales. Profesor de tiempo completo, Facultad de Derecho, Universidad Cooperativa de Colombia, sede Cali, Colombia. Correo electrónico: fernando.charriag@campusucc.edu.co
}

Recibido: 29 de octubre del 2016 Aprobado: 11 de noviembre del 2016

Cómo citar este artículo: Fernando CharriaGarcía. El concepto de cultura del derecho de autor. DIXI 25. Enero 2017. Págs. 71-81. doi: http://dx.doi.org/10.16925/di.v19i25.1822

\section{Resumen}

Propósito: el presente artículo efectúa una elaboración conceptual sobre la cultura del derecho de autor, para lo cual se realiza un acercamiento al concepto del derecho de autor, así como las dimensiones y teorías que lo soportan, a fin de determinar lo que es una obra y los derechos que le corresponden. Descripción: la investigación es una aproximación al concepto de cultura política que ha propiciado explicación a los valores, sentimientos y creencias del comportamiento político de las personas. Punto de vista: el autor se acoge a su experiencia en el campo del derecho, los estudios políticos, las artes y su enseñanza, para hacer una propuesta novedosa. Conclusiones: los anteriores conceptos son insumos estructurales de la propuesta que el autor hace sobre la necesidad de la elaboración del concepto de cultura del derecho de autor, con el fin de propiciar la realización de investigaciones que muestren la importancia que pueden tener los valores, los sentimientos y los conocimientos de las personas con respecto al derecho de autor, y con ello impactar políticas públicas en ese campo jurídico.

Palabras clave: cultura del derecho de autor, cultura política, derechos de autor, derechos humanos, obra. 


\title{
The concept of copyright culture
}

\begin{abstract}
Purpose: this article contains a conceptual elaboration on the copyright culture, for which an approach is made to the concept of copyright, as well as the dimensions and theories that support it, in order to determine what a work is and its corresponding rights. Description: the research is an approximation to the concept of political culture that has favored an explanation for the values, feelings and beliefs of people's political behavior. Point of view: the author uses his experience in the field of law, political studies, arts and their teaching, to make a novel proposal. Conclusions: the above concepts are structural inputs of the author's proposal on the need to elaborate the concept of copyright culture, in order to promote research that shows the importance of people's values, feelings and awareness with respect to copyright, and thus impact public policies in this legal field.
\end{abstract}

Keywords: copyright culture, political culture, copyright, human rights, work.

\section{O conceito de cultura do direito autoral}

\section{Resumo}

Propósito: este artigo realiza uma elaboração conceitual sobre a cultura do direito autoral; para isso, faz uma aproximação ao conceito do direito autoral bem como às dimensões e às teorias que o suportam a fim de determinar o que é uma obra $\mathrm{e}$ os direitos que correspondem a ela. Descrição: esta pesquisa é uma aproximação ao conceito de cultura política que gerou explicação aos valores, aos sentimentos e às crenças do comportamento político das pessoas. Ponto de vista: o autor recorre à sua experiência no campo do direito, aos estudos políticos, às artes e a seu ensino para fazer uma proposta inovadora. Conclusões: os anteriores conceitos são insumos estruturais da proposta que o autor faz sobre a necessidade da elaboração do conceito de cultura do direito autoral para propiciar a realização de pesquisas que mostrem a importância que podem ter os valores, os sentimentos e o conhecimento das pessoas a respeito do direito autoral e, com isso, impactar políticas públicas nesse campo jurídico.

Palavras-chave: cultura do direito autoral, cultura política, direitos autorais, direitos humanos, obra. 


\section{Propósito}

Las investigaciones jurídicas tienen una gran tendencia a buscar los aspectos de la normatividad jurídica como elementos a ser escrutados, y, en determinados casos, tal indagación puede adquirir algunos matices en torno a la eficacia, eficiencia y efectividad de las normas en estudio, lo cual requiere asumir unas indagaciones más precisas, como en el caso del contexto colombiano. Esto en lo relativo al lenguaje usado en las normas, su estructura y su andamiaje gramatical, junto con los aspectos relacionados de orden económico que supone la implementación de la norma, y los aspectos de recursos físicos y de personal que le son imprescindibles. Es importante también indagar ex-ante y ex-post, con el propósito de lograr entrever su impacto y sopesar debidamente la norma en estudio. No obstante, también es cierto que son relativamente pocos los acercamientos con énfasis etnográficos o propiamente sociojurídicos que se realizan.

En el campo del derecho de autor, en el cual el autor de este artículo se ha desempañado, desde vieja data se ha tenido la inquietud de indagar en relación con la manera en que las personas se relacionan con la norma y más allá de la norma, con lo que ella significa, su conocimiento, sus afectos y las valoraciones que suscita.

La razón para esto es porque con ello se puede lograr un acercamiento de mayor profundidad que permita incidir de manera más clara y estructurada en la construcción del sentido de orden que se pretende en el campo en estudio, generando una respuesta que brindará insumos fundamentales para iniciar acciones tendientes a incrementar su observancia, o construir herramientas que permitan proponer cambios que faciliten la observancia de dicho orden normativo.

En mis estudios de maestría en Estudios Políticos, encontré que lo que se conoce en dicho campo como "cultura política", está en la base de todo el andamiaje de lo que las personas entienden, sienten y valoran respecto de lo político; base sobre la cual se levanta todo el "edificio" de la idea de lo político y del poder que lo atraviesa, con lo cual, eventualmente, se podría incidir en las directrices o elementos que la componen, para así afectar las tendencias y facilitar la construcción del sistema político que se pretenda. Este fue el tema de mi tesis de grado hecha con dos compañeros más.

Tal vez unos tres años más tarde, hacia 1996, y pensando en relación con el derecho de autor, me pareció posible la idea de adaptar el concepto de cultura política al derecho de autor.
La idea cobró paulatinamente fuerza, hasta el punto de considerar que dicha idea permitiría hacer no solo una adaptación al campo jurídico específico, sino que facilitaría herramientas para incidir - a posteriori- de manera más clara y directa sobre el mismo, haciendo gala de las políticas públicas, entendiendo también el natural servicio y colaboración que las ciencias sociales se pueden prestar entre sí.

Aquí expondremos entonces dicha adaptación que hemos denominado "cultura del derecho de autor".

\section{DESCRIPCIÓN}

En este apartado se lleva a cabo una presentación de los referentes que nos interesan y son fundamento para la construcción conceptual que se propone.

\section{A. El derecho de autor}

El surgimiento del derecho de autor tiene un largo proceso histórico, muy ligado a los desarrollos técnicos y tecnológicos sobre la producción intelectual del ser humano.

De tiempo atrás, el respeto por la producción intelectual de una persona era reconocido no solo en los países orientales, sino también en los países latinos. ${ }^{1}$ Esto empezó a tener un mayor impacto precisamente con el desarrollo que tuvieron los medios de comunicación en la transición de la Edad Media, sobre todo los que surgieron por la aparición de la imprenta inventada por Gutenberg en el siglo xv, lo que permitió en gran medida la difusión de cierto tipo de obras. Esto propició también el surgimiento de muchas falsificaciones e imitaciones de dichas obras. Así, pues, el origen del derecho de autor está en relación con la lucha contra la piratería, ${ }^{2}$ por motivos de contenido patrimonial, dado el perjuicio económico que ocasionaba, pero también en lo que concierne a los escritos religiosos, pues existía una gran preocupación por controlar el surgimiento de alteraciones, variaciones y mutilaciones de las obras fundamentales de este campo.

Las primeras formas de protección consistieron en una especie de monopolios para el ejercicio del oficio de impresor con respecto a un determinado

1. Véase Fernando Charria García. Derechos de Autor en Colombia. Editorial Instituto Departamental de Bellas Artes. (2001). 2. Véase Ulrich Uchtenhagen. Introducción. Historia DEL DeREcho de Autor y los Derechos Conexos. Curso Regional de la ompi sobre Derecho de Autor y Derechos Conexos para PaíSEs de América Latina. (1997). Pág. 8. 
territorio (ciudad, reino, Estado o país), a lo que se le dio el nombre de "privilegio", el cual tenía por lo general una limitación de duración en el tiempo. Estos privilegios les fueron otorgados, en principio, a los editores y a los impresores. Posteriormente, la protección dio un giro en Inglaterra en favor de los autores con el llamado Estatuto de la Reina Ana de 1709, que fue promulgado en 1710, el cual terminó con el monopolio que había adquirido la Compañía de Impresores de Inglaterra en 1557 sobre la publicación de las obras en ese reino.

Con los procesos revolucionarios de Francia que abolieron los privilegios, se generó el sustrato para los decretos 1791 y 1793, que reconocieron a los autores los derechos de autorizar o prohibir la representación y la reproducción de sus obras, ${ }^{3}$ a los cuales se sumaron importantes desarrollos por la vía jurisprudencial sobre la consideración personal del autor, todo a partir de 1814.

Otro importante aporte se hizo desde Alemania, pues se dio un duro debate que permitió distinguir los dos aspectos de la protección de la personalidad en el derecho de autor: derecho moral y derecho patrimonial, y así usar el término de "derecho de autor" mucho mejor que el de derecho de bienes inmateriales, entre otros aspectos.

Estos desarrollos permitieron la creación del Convenio de Berna para la Protección de las Obras Literarias y Artísticas de 1886.

De igual manera, los avances continuaron hasta la creación, en el Convenio de Estocolmo del 14 de julio de 1967, de la Organización Mundial de la Propiedad Intelectual (омPI), la cual establece, en su artículo 2, las disciplinas que comprenden la propiedad intelectual y los derechos que las protegen, en relación con:

- Las obras literarias, artísticas y científicas.

- Las interpretaciones de los artistas intérpretes y las ejecuciones de los artistas ejecutantes, los fonogramas y las emisiones de radiodifusión.

- Las invenciones en todos los campos de la actividad humana.

- Los descubrimientos científicos.

- Los dibujos y modelos industriales.

- Las marcas de fábrica, de comercio y de servicio, así como los nombres y denominaciones comerciales.

- La protección contra la competencia desleal.

- Y todos los demás derechos relativos a la actividad intelectual en los terrenos industrial, científico, literario y artístico.

\footnotetext{
3. Véase Alfredo Vega. Manual de Derecho de Autor. Dirección Nacional de Derecho de Autor, Unidad Administrativa Especial, Ministerio del Interior y de Justicia. (2010).
}

Todos los anteriores son derechos llamados de "propiedad intelectual", aunque no todos tienen por objeto bienes inmateriales, ni sean reconocidos por razón de la creación intelectual de las personas. Así las obras literarias, artísticas y científicas son protegidas por el derecho de autor. Las interpretaciones y ejecuciones de los artistas intérpretes o ejecutantes, las fijaciones fonográficas y las emisiones de radiodifusión son protegidas por los derechos conexos. Las invenciones de nuevos productos están protegidas por los derechos de patentes. Los descubrimientos científicos están protegidos por los derechos de vincular públicamente los nombres de los investigadores a sus productos, y a obtener beneficios económicos por el aprovechamiento de tales descubrimientos. Los dibujos y modelos industriales son objeto de protección del derecho de dibujos y modelos industriales o también llamado "diseños industriales". Las marcas, las designaciones comerciales, los signos distintivos y las denominaciones de origen son protegidos por el derecho marcario o derecho de marca. La protección contra los actos de competencia desleal en materia comercial e industrial, se encuentran bajo el paraguas de protección de los derechos que regulan la competencia desleal.

El derecho de autor es entendido, entonces, como una rama del derecho cuya función es la protección de toda aquella producción humana, fruto de su ingenio, expresada de manera perceptible y susceptible de reproducirse por cualquier medio conocido o por conocer, que sea original, esto es, que tenga la impronta de su autor o autores y no sea una mera copia de otras ya existentes. Asimismo, el derecho de autor se despliega en la protección de los derechos morales de carácter personal, y en principio de duración ilimitada, y los derechos patrimoniales de carácter patrimonial con una limitada duración, en razón de la tensión-distensión entre los beneficios a que tiene derecho el autor, de una parte, y de otra, los beneficios a que tiene derecho la humanidad, todos como resultantes de la obra. En los países de tradición jurídica de derecho continental o romano-germánico, además de la expresión "derecho de autor", se utiliza la de propiedad literaria y artística, así como también la de propiedad intelectual.

Como hoy se conoce, los debates con relación a las facultades que constituyen el contenido del derecho de autor fueron causantes de la dificultad para determinar la naturaleza jurídica de este derecho. Así, es posible, hoy por hoy, hablar de varias teorías que dan soporte al concepto de naturaleza del derecho de autor. 


\section{Teoría del derecho de propiedad}

Esta teoría surge de la idea contenida en el preámbulo de la Ley del Estado de Massachusetts de 1789, marzo 17, en la cual se indica: "No existe propiedad más peculiar para el hombre que la que es producto de la labor de su mente". Este referente, así como otros, tales como los casos del Decreto 13 de enero 19 de 1791, y el del 19 del 14 de julio de 1793, en Francia, consagraron el reconocimiento del derecho de propiedad de la obra en cabeza del autor de la misma.

Otro debate se debió realizar en relación con la pertenencia o no del derecho de autor al campo del derecho de dominio sobre las cosas materiales, pues el derecho de autor plantea importantes diferencias, tales como la de que este último se ejerce sobre una producción intelectual y no sobre una cosa material. Por esto es que se debe entender que el derecho de autor surge con la mera creación de la obra y no en razón del cumplimiento de formalidades exigibles para aquellos derechos del dominio de cosas o in rem, pero además, es imposible adquirir este derecho de autor por vía de la prescripción. La duración del derecho moral de autor es ilimitada, y la del derecho patrimonial de autor es, por el contrario limitada. El régimen de coautoría es notablemente diferente al del régimen del condominio; el derecho moral de autor tiene como una de sus características la inalienabilidad, cosa contraria a la naturaleza del derecho de dominio. No es posible, por tanto, en el derecho de autor generar una transferencia plena del mismo, pues siempre estará ligada una parte de sus derechos a su autor.

En el desarrollo del derecho de autor durante el siglo XIX se configuraron dos tendencias: la de los dualistas, quienes separan las facultades del derecho de autor en morales y patrimoniales; y la de los monistas, quienes plantean que tal separación es ficticia, pues el derecho de autor es uno solo, dicen, mas se desdobla en dos facultades.

\section{Teoría del derecho sobre bienes inmateriales}

Según la profesora Delia Lipszyc, la teoría del derecho sobre bienes inmateriales fue elaborada por Josef Kholer, ${ }^{5}$ quien proponía que la idea de dominio solo puede referirse a cosas materiales, y que ello no era así en relación con el derecho del creador, puesto que se

\footnotetext{
4. Véase Yolanda Reyes. Los Oficios de la Imaginación. Dirección Nacional de Derecho de Autor. (2005) Pág. 26.

5. $C f$. Delia Lipszic. Derecho de Autor y Derechos Conexos. UNESCO-CERLALC. (1993). Pág. 22.
}

trata de un derecho exclusivo sobre la obra considerada un bien inmaterial, con una valoración económica, y que por ello difería de la naturaleza del derecho de propiedad que se aplica a los asuntos materiales. Por esta razón tuvo que admitir una nueva categoría la del derecho sobre los bienes inmateriales-, pues señala Kholer que el derecho de autor tiene solo una naturaleza patrimonial, tanto por su origen histórico, como porque sus normas están dirigidas a la tutela de las facultades exclusivas de reproducción, ejecución, representación, recitación, interpretación, distribución etc., de la obra, mediante las cuales el autor asegura la posibilidad de vivir económicamente del fruto de su trabajo. Los otros derechos del autor según Kholer- no forman parte del derecho de autor, sino de otro campo diferente que está fuera del individuo, de carácter intangible, que constituye una expresión concreta del derecho general de la personalidad.

Esta separación excluyente fue muy criticada, pues se indica que las facultades de carácter personal del autor no surgen de la personalidad general de un ser humano, sino que surgen de manera especial de la personalidad del autor que crea una obra, siendo así parte esencial del derecho de autor.

Importa en todo caso de esta teoría, la atención puesta en la definición del objeto sobre el cual recaen los derechos de autor, lo que facilitó la comprensión de estos derechos.

\section{Teoría del derecho de la personalidad}

Si en los inicios fue Kant quien señalara que el derecho de autor era en realidad un verdadero derecho de la personalidad, pues el producto de la creatividad humana era el expresado en la obra, producto para el que como tal habría espacio a un verdadero derecho real, también el contenido inmaterial de la obra implicaba un derecho personal. ${ }^{6}$

Los desarrollos de esta concepción fueron propugnados por Gierke, para quien el objeto del derecho de autor es una obra intelectual que constituye una emanación de la personalidad de su autor, quien logró atrapar la idea para condensarla expresada en un lenguaje en la creación, dando como fruto su obra, con lo cual la parte preponderante del derecho de autor son aquellos que pretenden dar garantía a la personalidad del autor y solo asume el carácter patrimonial como facultades accesorias.

\footnotetext{
6. Cf. Jhonny Antonio Pabón Cadavid (2009). Aproximación a la historia del derecho de autor: antecedentes normativos. Revista LA Propiedad Inmaterial 13. Págs. 59-104.
} 


\section{Teoría del derecho personal-patrimonial}

Los seguidores de esta teoría sostienen que el derecho de autor tiene una particular naturaleza, toda vez que se radica en cabeza de la persona, así como posee facultades patrimoniales, pues se ejerce en una doble función de protección: de un lado, sobre los intereses de la personalidad, y de otro, sobre los intereses patrimoniales.

Esta teoría recoge las dos posturas, la personalista y la patrimonialista, de manera ecléctica.

\section{Teoría de los derechos intelectuales}

Esta concepción, seguida por autores como Picard, Mouchet y Radaelli, ${ }^{7}$ y Satanowsky, ${ }^{8}$ considera que más allá de la tradicional división tripartita de los derechos en derechos reales, derechos personales y obligaciones, era necesario proponer para los derechos de autor y a la propiedad industrial la idea de una nueva categoría: la de los derechos intelectuales. Esta clasificación atendería al objeto del derecho de autor que es la obra, y abre una nueva categoría jurídica a fin de no asimilar bienes materiales y bienes inmateriales, proponiendo que esta nueva categoría de bienes intelectuales acogería, tanto los elementos personales o morales del autor, como los elementos patrimoniales o económicos, fruto de la explotación de la obra.

\section{Teorías varias}

Existen otras muchas teorías sobre la naturaleza jurídica del derecho de autor, sustentada esta en la idea del derecho natural, del derecho a la no imitación, del derecho de clientela, del derecho del trabajo, del derecho personal de crédito o de los derechos de monopolio. En fin, todo esto que no deja de ser interesante, se sale de los márgenes del presente escrito.

También es preciso señalar que la protección dada a los autores con respecto a sus obras, ha logrado un escaño en la Declaración Universal de Derechos Humanos de 1948, adoptada y proclamada por la Asamblea General de las Naciones Unidas, en su resolución 217 A (III), de 10 de diciembre de 1948, la cual en su artículo 27, numeral 2, señala: “Toda persona tiene derecho a la protección de los intereses morales y materiales que le correspondan por razón de las

\footnotetext{
7. Cf. Carlos Mouchet y Sigfrido Radaelli. Los DERECHOS DEL ESCRITOR Y DEL ARTISTA. Sudamericana. (1957).

8. $C f$. Isidro Satanowsky. Derecho Intelectual. tea. (1954).
}

producciones científicas, literarias o artísticas de que sea autora".

Esta inclusión en la Declaración Universal de Derechos Humanos, hace que tales derechos expresados como la protección a los intereses morales y materiales, adquiera un carácter de base mínima fundamental en la construcción de un ser humano digno, entendiendo la dignidad humana en el sentido de que el ser humano no debe ser medio para nada, sino un verdadero fin en sí mismo. Se complementa lo anterior con la idea de mínimos, que es propia de tal declaración, en el entendido de que los derechos allí consagrados, tanto los civiles, como los políticos y los sociales, económicos y culturales, son mínimos básicos, lo cual supone la posibilidad a futuro de un incremento de ese mínimo, consagrando otros derechos que deben coadyuvar a la dignidad del ser humano.

Otra idea que cabe resaltarse es que "los derechos humanos se han construido, principalmente, a las amenazas de la libertad y el patrimonio de los individuos. Estas exigencias se articulan a través de la limitación del poder y de su intervención protectora, es decir, abstenerse de vulnerar e intervenir a fin de proteger". ${ }^{10}$

No obsta señalar que, en todo caso, estos derechos consagrados en la declaración a la que se hace referencia, también son fundamentales para la construcción de democracia, y allí el papel de los derechos de autor contribuye de igual forma con esta construcción, puesto que una de las características estructurales de la democracia es el respeto por el otro, y ese otro, que no solo es indispensable en la construcción de nosotros como sujetos, sino que es indispensable para que nosotros nos construyamos culturalmente como humanos, implica un sujeto que va más allá de su piel, implica un sujeto que se entiende en sus producciones intelectuales, de las cuales el lenguaje da cuenta de maneras curiosas en su verbalización. Esto en cuanto puede referirse al hecho de "tener a Picasso", por ejemplo, cuando se posee una obra de un autor, cuando lo que realmente se tiene no es al autor, sino a una obra del mismo, es decir, "tener un Picasso".

Son, pues, los derechos de autor ostentadores de un derecho inalienable del ser humano y fundamental en la construcción de democracia.

\footnotetext{
9. Véase A.G. Resolución 217 A (III). Artículo 27, numeral 2. (Diciembre 10 de 1948).

10. Véase Carlos R. Asúnsolo-Morales. Los derechos humanos como límites al poder público y privado: otra vía de fundamentación. Revista DIXI 22. Diciembre 2015. Pág. 95.
} 


\section{B. Cultura política}

Los antecedentes de lo que hoy conocemos como cultura política van de la mano con los inicios mismos de la ciencia política, pues nació a propósito del derrumbe de la democracia en Weimar y el surgimiento del nazismo, generando con ello un esfuerzo intelectual por entender dicho fenómeno. Esto logró inquietar de manera particular a las ciencias sociales norteamericanas y alemanas, pues la no verificación de la teoría liberal y marxista frente a dicho derrumbamiento, fue el caldo de cultivo propicio para la construcción de la cultura política en su versión moderna. ${ }^{11}$

El centro de la idea de cultura política es el énfasis en la importancia de los valores, sentimientos y creencias, y con ello en la explicación del comportamiento político de las personas, pues no son simples reflexiones sobre la estructura política, ni son susceptibles de reducirse a la posibilidad de que el individuo haga una elección racional, desconociendo de esta manera el influjo que sobre este individuo tiene lo social.

El concepto de cultura política adquiere cierta independencia conceptual a partir de los años sesenta gracias, entre otros, a los trabajos efectuados por Gabriel Almond y Sidney Verba, quienes efectuaron algunas de las primeras definiciones, con la intención de tender un puente entre las explicaciones conductivistas o behavioristas, y las sociológicas o macroanalíticas del comportamiento humano relacionado con lo político.

Esta expresión de cultura política designa "un conjunto de actitudes, normas y creencias compartidas más o menos ampliamente por los miembros de una determinada unidad social y que tienen como objeto, fenómenos políticos". ${ }^{12}$

Asimismo, Sani señala que forman parte de la cultura política de una sociedad "los conocimientos, o mejor aún, la distribución entre los individuos que la componen, de los conocimientos relativos a instituciones, a la práctica política o a fuerzas políticas que operan en un contexto determinado", así como "las orientaciones más o menos difundidas, por ejemplo: la indiferencia, el cinismo, la rigidez, el dogmatismo, la adhesión, la tolerancia, hacia fuerzas políticas distintas de la propia"; también las normas, "nociones

11. Véase Gabriel Almond. El estudio de la cultura política. Revista DE CIENCIA POLíticA 10(2). Pág. 77-89. Disponible en 1988.https:// repositorio.uc.cl/bitstream/handle/11534/10833/000129200. pdf? sequence $=1$ \&is Allowed $=y$

12. Véase Giacomo Sani. Cultura Política. Diccionario de Ciencia Política. Pág. 470 de Derecho y de Deber que los ciudadanos comunes poseen para participar en la vida política, su obligación para aceptar decisiones de la mayoría, su inclusión o exclusión de ciertos procesos políticos".

En general, la teoría de la cultura política se define con base en cuatro aspectos: 1 . La cultura política es el conjunto de orientaciones subjetivas hacia la política de los miembros de una nacionalidad, o subconjunto de estos en la misma nacionalidad; 2 . La cultura política posee componentes cognoscitivos, afectivos y evaluativos; 3 . El contenido de la cultura política es el resultado de la socialización infantil, la educación y la exposición a los medios de comunicación y las experiencias durante la edad adulta con respecto a la acción gubernamental, social y económica; y 4 . La cultura política afecta la estructura política y gubernamental y el desempeño la restringe, pero sin que por ello se determine, es decir, que existe una importante relación entre estructura, cultura y desempeño.

Las orientaciones, como ya hemos indicado, se presentan con tres componentes básicos: cognoscitivos, afectivos y valorativos.

La orientación cognoscitiva está representada por el conjunto de conocimientos y creencias relativas al sistema político, a las funciones de los diferentes estamentos y a los titulares de esas funciones. La orientación afectiva representa el conjunto de sentimientos albergados en relación con el sistema y con su estructura. Por último, las orientaciones valorativas involucran juicios y opiniones sobre fenómenos políticos, y requieren, a su vez, la combinación de informaciones, sentimientos y criterios de valoración.

Estas tres orientaciones básicas permitieron que en sus combinaciones proporcionaran arquetipos de la cultura política, lo que Almond y Verba denominaron "orientación provinciana o parroquial", "orientación al sujeto o subordinación”, y "orientación a la participación".

En el primer caso de orientación parroquial, se indica que existe un bajo nivel de conocimientos, sentimientos y criterios en relación con los aspectos evaluados de las orientaciones, presentándose en sociedades retrógradas y en sectores de sociedades modernas tales como los juveniles, femeninos y en poblaciones rurales; por tanto, característicos de sociedades no diferenciadas, o sociedades simples.

En el segundo caso de orientación de subordinación, tiene un bajo nivel de conocimientos e involucramiento, pero en relación con el conocimiento sobre el sistema político en el cual al actor político informado, está al tanto del rendimiento político del sistema, esto es, lo importante son las respuestas del sistema, pero 
no los aportes de los sujetos particulares al sistema. Es propio de sistemas en los que la burocracia es benévola y cumple un papel principal, o en los regímenes totalitarios en los que existen rituales de participación masiva de manera permanente.

En el tercer caso de las orientaciones de participación, se caracteriza por el conocimiento y el involucramiento político por parte de los sujetos en los aspectos de las orientaciones ya descritos.

Por ello, surgen los conceptos de adhesión al sistema, alienación al sistema (como una actitud de hostilidad), y apatía al sistema (como una actitud de indiferencia).

Es importante señalar que la cultura política ha sido objeto de críticas que han surgido desde cuatro perspectivas diferentes. La primera crítica le atribuye a la cultura política aspectos deterministas, pues supone que la socialización política produce actitudes políticas, esto es, la tendencia psicológica que alguien tiene respecto de algo o de alguien. Por tanto, son verdaderas predisposiciones que deben producir comportamientos políticos los que estarían en la base de la estructura política. En este sentido, los defensores de la cultura política reconocieron que la causalidad influía, tanto en las actitudes y estas en el comportamiento y en la estructura, como que la estructura y el comportamiento influían en las actitudes, con lo cual se trata de una crítica que evidencia poca fortaleza conceptual.

Una segunda crítica procede del marxismo, según la cual el cambio de actitudes proviene de una transformación de la estructura económica, de manera que en sana lógica marxista, el cambio se realiza de la estructura económica hacia las actitudes políticas, los comportamientos políticos y las estructuras políticas. Todo lo anterior implica concebir que las actitudes políticas tienen un contenido estructuralmente necesario, por lo cual estas no tienen un poder explicativo autónomo. Argumentación que ya no es expresada por los neomarxistas, quienes señalan que la política y el Estado presentan cierta autonomía, y que la etnia, la religión y la nacionalidad presentan un proceso de resocialización de alta dificultad.

Una tercera crítica señala que no es posible separar las actitudes de los comportamientos políticos, evitando con ello separar la dimensión psicológica de la conductual, lo que impide estudiar las relaciones entre pensamiento político y acción política, pues al unir actitudes y comportamiento alegando su inseparabilidad, se impide con esta diferenciación el estudio de las relaciones entre pensamiento y acción política.

Una cuarta crítica indica que la estructura y el comportamiento políticos pueden ser explicados realizando cálculos sobre el beneficio a corto plazo de los actores políticos. Es decir, la explicación hace caso omiso de valores, normas, sentimientos y aspectos cognoscitivos, pues la simple introducción de la elección racional es suficiente para dar explicación en cualquier situación política.

En todo caso, en la actualidad, la idea que persiste relativa a la cultura política no es la que se tuvo en los años cuarenta del siglo pasado, influida por ideas con una fuerte afectación por parte de los familiares, otras afectaciones generadas en épocas infantiles y de aquellas dominadas por el inconsciente, sino una teoría que pone en realce el ámbito cognoscitivo, las actitudes y las expectativas influidas por la estructura y desempeño del sistema político y económico. Se da cabida también a unos elementos más permanentes y estables como las creencias, los compromisos con valores y adhesiones, que inciden en el comportamiento político y en la posibilidad de las políticas públicas.

\section{Punto DE VISTA}

A continuación, se propone un punto de vista conceptual propio que supone la integración de las ciencias sociales del derecho y la politología, concretadas en el concepto de cultura del derecho de autor que se desarrolla a continuación.

\section{A. Cultura del derecho de autor}

La posibilidad de indagar desde un lugar que permita un acercamiento un poco más etnográfico en el campo del derecho de autor, se convierte en una necesidad importante, en la medida en que la preocupación que me asiste como una persona comprometida con el derecho de autor, como autor de un libro al respecto en Colombia ${ }^{13} \mathrm{y}$ abogado en ejercicio en este ámbito e investigador, es indagar en las personas su relación con esta normatividad.

Parte de la preocupación surge porque en el ámbito académico se vienen presentando inquietudes por el hecho de presentarse múltiples problemas relacionados con el plagio. Por otra parte, causa curiosidad e interés el hecho de que se haya tenido que ir hasta los más altos tribunales en Colombia, concretamente a la Corte Constitucional, para resolver un asunto que implicó una obra de arte y la supuesta violación de unos derechos de autor a una imagen,

13. Véase Fernando Charria García, supra, nota 1. 
así como otros derechos de injuria y calumnia supuestamente afectados por la obra De blanco porcelana. ${ }^{14}$ Otro tanto sucedió con la censura artística realizada en Bogotá en el Museo Santa Clara, a la obra de la artista María Eugenia Trujillo, lo cual nos acerca a despropósitos y vejaciones al derecho de autor de otros artistas, como fue el caso típico en Colombia de la maestra Débora Arango, artista antioqueña quien realizó en Medellín una exposición que fuera obstaculizada en 1939, de manera muy fuerte por las Damas de la Liga de la Decencia, o su exposición en el Teatro Colón de Bogotá en 1940, que fuera cerrada por órdenes del político Laureano Gómez, conservador de gran influencia en el país y quien posteriormente llegó a la presidencia de Colombia, a quien le parecían inmorales sus desnudos. Esto se junta fácilmente con lo que le sucediera en su exposición de Madrid, España, en 1955, realizada en el Instituto de Cultura Hispánica, que fue clausurada por el gobierno de aquel entonces en cabeza de Francisco Franco. ${ }^{15}$

Esta preocupación surgida hacia 1996, y luego afirmada cada vez más por el trabajo desempeñado en Bogotá en el Instituto Distrital de Cultura y Turismo entre el 2000 y el 2004 en el campo del derecho de autor, unido al hecho de haberme desempeñado como rector de una institución dedicada a la enseñanza de las artes en la ciudad donde resido, concretamente en el Instituto Departamental de Bellas Artes en Cali, Colombia, entre noviembre del 2010 y noviembre del 2013, así como en los hechos ya relatados, fueron razones suficientes para escribir el elemento conceptual que permitiera la posibilidad de realizar investigaciones, ya no documentales, sino más con un ingrediente o carácter etnográfico que en el derecho conocemos como "sociojurídicas".

Así, entonces, podríamos afirmar que en el ámbito de la doctrina jurídica no se ha encontrado inquietud alguna que permita hablar de la idea de cultura en relación con un aspecto específico del derecho que acoja la inquietud por unir aspectos afectivos, de creencias y valorativos, aunque hemos observado que sí es posible, desde la perspectiva filosófica, encontrar autores que hablen de la cultura del derecho, pero entendida esta como la idea que un sujeto puede tener, dado su saber, respecto del derecho en general, pero no sobre la cultura del derecho aplicada a algún

14. Se trata de la obra Blanco Porcelana de la artista Margarita Ariza. Véase la Sentencia T-15/2015.

15. Cf. Arcadia. Censura artística en el Museo Santa Clara. RevistaArCadia.com.Agosto19del2014.Disponibleenhttp://www. revistaarcadia.com/arte/articulo/censura-artistica-en-el-museosanta-clara/38432 ámbito específico del mismo, en el sentido de indagar por sus orientaciones, tal y como lo hicieran Almond y Verba respecto de lo político; al menos hasta ahora en las indagaciones efectuadas para poder estructurar esta investigación, no se ha encontrado.

Importa entonces pensar que es posible por procesos de analogía y aplicación de las experiencias de otras ciencias sociales, hacerlo con respecto a algún campo específico del derecho, para permitir efectuar acercamientos y análisis más completos que ayuden a generar respuestas mucho más precisas y aclarar el panorama en que viven las personas en relación con el ámbito que se habrá de indagar. Esto significa que aquellas preocupaciones que permitan indagar sobre las actitudes o predisposiciones que las personas tengan sobre el derecho de autor, también con relación a las normas y el conocimiento que de ellas se tenga, que va más allá de la mera información y las creencias compartidas por aquellas personas que son sujetos activos en la creación de obras artísticas o científicas, preocupaciones que deben permitir construcción de perspectivas sobre las protecciones o no que se les brinda a dichas obras, por cuanto desconocemos, a pesar de las indagaciones realizadas, que se haya planteado buscar explicaciones sobre la base de indagar a estas personas autoras respecto de los asuntos planteados.

Por supuesto, la intención de esta indagación no es llenar un mero prurito personal, sino construir una herramienta que nos ayude a ubicar perspectivas de relación con ese mundo del derecho de autor por parte de los autores, las autoridades y demás actores del proceso de creación y de la obra, su reproducción y difusión.

En tal sentido, se podría plantear como cultura del derecho de autor aquel conjunto integrado por las orientaciones cognoscitiva, afectiva y valorativa que las personas tienen respecto del derecho de autor. En este mundo del derecho de autor, caben no solo las normas, sino todo aquello que las personas autoras crean que conforman ese mundo, tal como los casos de las mismas normas, los libros y textos que hablan acerca del derecho de autor, las instituciones y personas garantes del mismo, en fin, todo aquello que perciban al respecto.

Por lo anterior, podemos entender por orientación cognoscitiva aquella representada por el conjunto de conocimientos y de creencias relativas al derecho de autor, a las funciones de los diferentes estamentos y a los titulares de esas funciones. Esto significa no solo lo que efectivamente es informado, sino también lo que ha sido debatido y, digámoslo así, cultivado, que permita una instancia superior a la mera información que constituye el conocimiento; pero también las creencias, esto 
es, si eso del derecho de autor sirve o creemos que es otra invención humana, o un artilugio para impedir el conocimiento, etc.

Por orientación afectiva se entiende el conjunto de sentimientos albergados en relación con el derecho de autor y su estructura. Esto significa sentimientos de cercanía o de lejanía, sentimientos de formar parte o de no formar parte, incluyendo sus estructuras, esto es, los lugares y formas de enseñanza del derecho de autor, los sistemas de protección y acceso a la administración de justicia, tanto de carácter nacional, como internacional, sus formas de organización por parte de los autores y los consumidores, entre otros.

$\mathrm{Y}$ por último, las orientaciones valorativas como aquello que involucra juicios y opiniones sobre fenómenos del derecho de autor, lo cual requiere, a su vez, la combinación de informaciones, sentimientos y criterios de valoración. Con esto se logra completar aquella complejidad de la que se habla cuando se refieren las orientaciones, puesto que se trata de una sola concepción que tiene varios componentes, pero en la que todos deben actuar a una.

Como resultado de ello, los conceptos de adhesión al derecho de autor, que supone un acercamiento al ámbito del derecho de autor, tanto a sus normas como a sus estructuras, a sus formas de enseñanza y a los espacios para su debida protección; el concepto de alienación al derecho de autor, que debe entenderse como una actitud de hostilidad respecto del derecho de autor, dadas sus consideraciones; $y$ por último, el concepto de apatía al derecho de autor, que supone una actitud de indiferencia frente al mismo y no trata de un rechazo, sino de una ausencia de importancia del mismo, se convierten en conceptos de gran importancia para caracterizar el tipo de orientación que se pretende indagar.

Lo anterior supone, además, la necesidad de realizar indagaciones teniendo en cuenta el factor económico, que puede ser representado en el sentido de que es muy posible que las respuestas dadas por una persona de clase social alta, sean diferentes a las de una de clase social media o baja, en razón a que el determinante de la economía está en relación con otro tipo de procesos y circunstancias que los envuelven y constituyen, como el caso del acceso a la educación de calidad, el acceso a bienes de información con soporte material o inmaterial (Internet, por ejemplo).

Lo anterior nos abre el espectro para realizar investigaciones, una de las cuales se encuentra en este momento en proceso. Para esto es imprescindible la construcción de herramientas que tengan en cuenta las orientaciones que se han mencionado, así como la clase social o el referente económico, todo lo cual estará determinado por el lugar donde se realice la investigación, no solo desde la perspectiva geográfica, sino de las instituciones y de los actores de la misma, pues ello tendrá incidencia en el tipo de herramienta y en los resultados que se recojan.

\section{Conclusiones}

El concepto aquí expresado muestra la viabilidad, la pertinencia y la utilidad para lo que fue creado. En este sentido, el concepto facilita la creación de herramientas estructuradas de investigación que respondan por las orientaciones, de manera que es posible el estudio específico con respecto a los conocimientos, las evaluaciones o valoraciones y los sentimientos que las personas indagadas puedan tener en relación con el derecho de autor.

La aplicación de este concepto tiene plena pertinencia, por cuanto en el curso de los años 2017 y 2018 , se pondrá a prueba en investigaciones en el campo de la propiedad intelectual en América Latina, y en aquellos otros lugares donde sea posible.

Los resultados de la aplicación investigativa habrán de convertirse en soporte estructural para nuevas políticas públicas nacionales o internacionales en el campo.

\section{REFERENCIAS}

A.G. Resolución 217 A (III). Artículo 27, numeral 2. (Diciembre 10 de 1948).

Alfredo Vega. Manual de Derecho de Autor. Dirección Nacional de Derecho de Autor, Unidad Administrativa Especial, Ministerio del Interior y de Justicia. (2010).

Arcadia. Censura artística en el Museo Santa Clara. RevisTAarCadia.com. Agosto 19 del 2014. Disponible en http://www.revistaarcadia.com/arte/articulo/censura-artistica-en-el-museo-santa-clara/38432

Carlos Mouchet y Sigfrido Radaelli. Los DERECHOS DEL ESCRITOR Y DEL ARTISTA. Sudamericana. (1957).

Carlos R. Asúnsolo-Morales. Los derechos humanos como límites al poder público y privado: otra vía de fundamentación. Revista DIXI 22. Diciembre 2015.

Delia Lipszic. Derecho de Autor y Derechos Conexos. UNESCO-CERLALC. (1993).

Fernando Charria García. Derechos de Autor en CoLомвiA. Editorial Instituto Departamental de Bellas Artes. (2001). 
Gabriel Almond. El estudio de la cultura política. REvisTA DE Ciencia Política 10(2). Pág. 77-89. 1988. Disponible en https://repositorio.uc.cl/bitstream/handle/11534/10833/000129200.pdf? sequence $=1$ \&isAllowed $=y$

Giacomo Sani. Cultura Política. Diccionario de Ciencia PolíticA.

Isidro Satanowsky. Derecho Intelectual. TeA. (1954).

Jhonny Antonio Pabón Cadavid (2009). Aproximación a la historia del derecho de autor: antecedentes normativos.
Revista La Propiedad Inmaterial 13. Págs. 55-104. Ley del Estado de Massachusetts de 1789. Marzo 17 de 1789. Ulrich Uchtenhagen. Introducción. Historia DEL Derecho de Autor y los Derechos Conexos. Curso RegioNAL de la ompi sobre Derecho de Autor y Derechos Conexos para países de América LATINA. (1997). Pág. 8.

Yolanda Reyes. Los Oficios DE LA Imaginación. Dirección Nacional de Derecho de Autor. (2005) Pág. 26. 